\title{
Chapter 17 \\ Novel Modalities to Diagnose and Prevent Compartment Syndrome
}

\author{
Andrew H. Schmidt
}

\section{Background}

Acute compartment syndrome (ACS) of an extremity following trauma remains a diagnostic and therapeutic enigma. This phenomenon is because the clinical signs and symptoms of ACS are variable and nonspecific, and there are multiple causes of ACS after trauma (Table 17.1). Furthermore, the pathophysiology of ACS is poorly understood, which leads to confusion regarding the ideal diagnostic strategy. For decades now, ACS has been considered to be a pressure-related pathophysiologic

Table 17.1 Causes of acute compartment syndrome

\begin{tabular}{l}
\hline Causes of acute compartment syndrome after trauma \\
\hline 1. Accumulation of mass within the muscle compartment \\
\hline Bleeding \\
\hline Edema \\
\hline Tissue injury caused by direct trauma \\
\hline Inflammation and/or toxins \\
\hline Postischemic reperfusion \\
\hline Insertion of internal fixation hardware \\
\hline 2. Reduction of compartment volume \\
External compression from bandages, splints, casts \\
\hline Changes in the limb length related to fracture treatment \\
\hline External fixation \\
\hline Traction \\
\hline Internal fixation
\end{tabular}

\footnotetext{
A. H. Schmidt $(\square)$

University of Minnesota, Minneapolis, MN, USA

Department of Orthopedic Surgery, Hennepin Healthcare, Minneapolis, MN, USA

e-mail: schmi115@umn.edu 
process, with diagnostic recommendations largely based on pressure thresholds either the absolute intracompartment pressure (ICP) or some measure of the perfusion pressure (defined as the difference between ICP and the patient's blood pressure). However, defining a single pressure threshold that accurately defines when fasciotomy is needed has not proven possible, and multiple studies suggest that clinical use of such thresholds leads to overtreatment [1-3].

Current treatment of ACS is surgical fasciotomy, which if not performed before the onset of cellular necrosis results in intractable pain, muscle fibrosis and contracture, and sensory deficits. Fasciotomy restores perfusion by increasing compartment volume so that the ICP falls, allowing tissue perfusion to be restored in viable vascular beds. This benefit is consistent with the traditional view of ACS as a "pressure problem." However, it is more appropriate to consider ACS as a metabolic problem, and both diagnostic and therapeutic strategies aimed at assessing cellular viability and reversing metabolic abnormalities may not only make our diagnosis of ACS more precise but also provide opportunities for less invasive ways to prevent ACS and mitigate the sequelae of ACS when it does occur.

\section{New Concepts in ACS Pathophysiology}

Compartment syndrome has been considered to be the result of tissue ischemia caused by decreased perfusion of the limb from elevated ICP, which may occur from any one of the several mechanisms: arterial spasm, collapse of arterioles, or collapse of the venous system. However, a recent series of publications by researchers in London, Ontario, Canada, has shown that ACS is accompanied by both a local and a systemic inflammatory response that may play a significant role in the pathogenesis of ACS and the resultant tissue damage [4-9]. This knowledge "opens the door" to using inflammatory mediators as potential biomarkers for understanding the progression or resolution of ACS and anti-inflammatory therapies as either primary or adjunctive medical therapy for ACS.

In a series of animal studies [4-9], the relationship between elevated ICP and the inflammatory response on skeletal muscle microcirculation and cell viability was measured in rats using intravital videomicroscopy. In one study, the level of tissue injury in an experimental compartment syndrome was compared between normal rats and those with neutropenia induced by injection of high-dose cyclophosphamide [5]. Muscle cellular injury was reduced more than 50\% in neutropenic compared to healthy rats. Furthermore, the control rats had higher tissue injury $(23.0 \% \pm 4.0 \%$ of cells $)$ compared to the neutropenic group $(7.0 \% \pm 1.0 \%$ of cells $)$ after 90 minutes of elevated ICP $(p=0.00005)$ [5].

In a second study using a similar experimental model, local and systemic cytokine activation was measured and showed that animals with ACS have a systemic inflammatory response in addition to the local injury [4]. Remote organ damage was measured in the liver using intravital videomicroscopy techniques. Leukocyte activation, increased serum TNF- $\alpha$ levels, and necrotic hepatocytes were noted after 2 hours of ACS, all indicating systemic effects from ACS induced in one limb. 
These same researchers have also demonstrated that attenuation of the inflammatory response may be beneficial in cases of ACS. Manjoo et al. [9] studied the effect of indomethacin on the level of tissue injury measured in rats with induced elevations in ICP for 45 or 90 minutes. Tissue injury was reduced almost to baseline levels in rats that were pretreated with indomethacin; a smaller benefit was noted when indomethacin administration was delayed [9]. In another study using the same model, treatment with a different anti-inflammatory molecule (CO-releasing molecule 3) led to statistically significant improvement in the number of continuously perfused capillaries, resulted in decreased tissue injury, reversed the ACS-associated increase in TNF- $\alpha$, and reduced leukocyte adherence [6].

This series of papers indicate that ACS is accompanied by a substantial inflammatory response in the involved limb that also affects other organ systems. This information suggests that mitigation of the tissue injury caused by ACS may be possible by decreasing inflammation. Further work to determine the benefits of inflammatory modulation in a larger animal model is necessary, followed by clinical research in humans. The ideal therapeutic agent, mode of delivery, and timing are all unknown questions at this time.

\section{New Diagnostic Modalities}

The traditional diagnosis of ACS has been based on the clinical examination, supplemented when needed by intracompartment pressure measurement. Newer diagnostic approaches to ACS include near-infrared spectroscopy (NIRS), biomarker analysis, and radiofrequency identification (RFID) implants. These new approaches are discussed in the following paragraphs.

\section{NIRS}

In the context of measuring tissue perfusion in possible cases of ACS, NIRS uses differential light reflection and absorption to estimate the proportion of hemoglobin saturated with oxygen approximately $2-3 \mathrm{~cm}$ below the surface of the skin [10-14]. The depth of tissue interrogation is determined by the distance between the light source and the receptor.

The potential use of NIRS to diagnose ACS is supported by preclinical studies. In 1999, Garr et al. induced compartment syndrome in anesthetized pigs and showed that NIRS was a better predictor of neuromuscular dysfunction than compartment perfusion pressure [10]. However, its clinical use has shown mixed success. A case report published in 2011 outlined the benefits of using NIRS in three cases of lower extremity ACS [13]. NIRS differentiated between adequately perfused lateral compartments and poorly perfused deep posterior compartments of one patient with ACS. In another unresponsive patient diagnosed with ACS on clinical means, NIRS was able to detect perfusion deficits. In a third patient, NIRS demonstrated changes 
in muscle perfusion due to anesthetic induction within seconds [13]. However, also in 2011, Bariteau et al. described seven patients with a clinical diagnosis of ACS who had oxyhemoglobin saturation $\left(\mathrm{rSO}_{2}\right)$ and ICP values measured in each compartment of the affected lower extremity before undergoing fasciotomy. No statistically significant association was observed between $\mathrm{rSO}_{2}$ and ICP or perfusion pressure [11].

Many questions remain about the clinical use of NIRS in traumatized limbs for diagnosing ACS, such as the ideal penetration depth, the effect of skin pigmentation and subcutaneous fat, the effect of skin abrasion or degloving, and subcutaneous or intramuscular bleeding. It has been demonstrated that injured limbs have a hyperemic response to injury, resulting in increased $\mathrm{rSO}_{2}$ values [14].

One large theoretic advantage of NIRS is its potential ability to demonstrate changes in tissue perfusion corresponding with the onset of ACS. However, continuous NIRS has not been as well studied. Two recent papers have reported on continuous NIRS in a clinical setting. Shuler et al. [15] reported on 109 patients who had NIRS recording of the tissue perfusion in all four leg compartments of both legs (anterior, lateral, superficial posterior, and deep posterior): 86 had unilateral leg injuries while 23 did not. Mean NIRS values were between $72 \%$ and $78 \%$ in injured legs, between $69 \%$ and $72 \%$ in uninjured legs, and between $71 \%$ and $73 \%$ in bilaterally uninjured legs. NIRS values were typically $>3 \%$ higher in injured limbs without ACS than in uninjured compartments. In contrast, all seven limbs with clinically diagnosed ACS had at least one compartment where NIRS values were $3 \%$ or more below an uninjured control compartment [15].

One problem with NIRS that may be even more of an issue when using NIRS in a continuous manner is the reliability of data capture. Shuler et al. noted that "Missing data were encountered in many instances" [15]. Schmidt et al. [16] used blinded continuous NIRS and blinded continuous ICP monitoring in 191 patients with leg injuries [16]. Data capture was unreliable with NIRS, with simultaneous data available from both injured and control limbs just $9 \%$ of the expected time, comparted to $88 \%$ of the expected time with continuous ICP [16]. At this time, it is not clear whether these datacapture issues with NIRS represent clinically immature technology that needs further development for this purpose or represent fundamental problems using NIRS to measure soft tissue oxygenation when that same tissue has had traumatic injury [16].

\section{Biomarkers}

Markers of muscle injury or metabolic disturbances such as acidosis may be measured systemically or locally.

Odland et al. have applied tissue ultrafiltration (TUF) catheters to the problem of diagnosing and treating acute compartment syndrome [17, 18]. TUF uses small diameter, flexible, hollow fiber catheters connected to suction in order to remove interstitial fluid, which may contain biomarkers of injury and contribute volume to the muscle compartment. The possible therapeutic benefits of TUF are discussed later in this chapter. In their study, serum and ultrafiltrate levels of creatine kinase 
and lactate dehydrogenase were measured hourly. The study demonstrated that biomarker concentrations were up to 80-fold higher in ultrafiltrate than in serum.

A promising metabolic marker for ACS is tissue $\mathrm{pH}$, as measured by intracompartment probes. Basic science studies done at the University of Aberdeen in Scotland have revealed a correlation between local $\mathrm{pH}$ and depletion of high-energy phosphate stores [personal communication, Alan Johnstone 2018]. These same researchers have demonstrated that in a small study continuous $\mathrm{pH}$ measurement may be better than continuous pressure measurement at diagnosing ACS [19]. Further research is ongoing that should better define the role of $\mathrm{pH}$ monitoring on this setting.

\section{RFID Chips in Diagnosing Compartment Syndrome}

Current methods of diagnosing ACS rely on catheters or sensors that must remain attached to the patient and are therefore subject to inadvertent removal or may interfere with aspects of patient care, such as transportation, imaging, splinting, or the performance of surgical procedures. An "always-on" device that can be applied in a minimally or noninvasive manner and that does not interfere with transportation or other care of the patient would be a significant advance. New sensor technology that utilizes radiofrequency electromagnetic fields (radiofrequency ID tags or RFIDs) to transfer data exists and is beginning to be used for monitoring physiological systems [20]. Such devices do require a battery and are powered externally by the very fields used to read their data. RFID pressure sensors have been developed and used for compartment monitoring [20].

\section{Potential Therapeutic Advances in Treating ACS}

In at-risk or early phases of compartment syndrome, before tissue necrosis has started to occur, other means of improving tissue perfusion and oxygenation may exist. One method would be to increase the tolerance of the muscle to ischemia. Other areas of theoretical interest include methods to decrease intramuscular pressure (tissue ultrafiltration, foot pumps, mannitol, and diuretics), improving tissue oxygenation, mitigating the effects of ischemia (free-radical scavengers and other pharmacologic measures), and small-volume resuscitation with hypertonic saline.

\section{Tissue Ultrafiltration}

Compartment syndrome is associated with accumulation of mass within a muscle compartment of relatively fixed volume, which is what results in the progressive increase in intramuscular pressure, which eventually sets in motion the vascular 
embarrassment and associated metabolic changes that result in tissue death. By removing even small amounts of fluid, loss of the associated fluid mass within the compartment may decrease compartment pressures. In an animal study, the potential benefits of tissue ultrafiltration (TUF) were demonstrated [18]. A bilateral infusion model of compartment syndrome in the pig hindlimb was used; each animal had one control limb and one treatment limb. Muscle pressure was measured with an indwelling catheter. Three TUF catheters were inserted into the anterior compartment of each limb. The catheters in the treatment limb were connected to negative pressure suction. The ICP pressure was lower in the treated limbs, and the muscle histology was more normal in the treated limbs compared to the control legs [18]. This study was followed up with clinical research on TUF in a small human trial. A pilot study demonstrated the feasibility of TUF in ten patients with tibial shaft fractures treated with nailing [17]. A small-randomized clinical trial of TUF versus pressure monitoring alone was also performed [21]. Fourteen patients were randomized to two groups: one used TUF plus monitoring while the other was monitored alone. Anterior and deep posterior pressures were measured for 24 hours in all patients. Outcome measures between the two catheters were compared using T-tests. The mean intramuscular pressure was smaller than in the control group in both the anterior and posterior compartments. Because of insufficient power, these differences were not statistically significant. Interestingly, two patients in the control catheter group developed compartment syndrome -8 and 16 hours after the initial surgery and catheter insertion. No compartment syndromes were diagnosed in the treatment group.

\section{Hypothermia}

Tissue cooling has been used to improve tissue viability in transplant and replant surgery and is of known value in treating soft tissue injury. Sanders and colleagues, using a well-developed rat model (as described in the "New Concepts in ACS Pathophysiology" section of this chapter), showed that hypothermia to $25{ }^{\circ} \mathrm{C}$ reduced tissue damage score by $50 \%$ and also seemed to attenuate the inflammatory response [22]. Although this information is interesting, at this time the role of induced hypothermia for treating ACS in humans is not defined.

\section{Conclusion}

New concepts and ideas about the pathophysiology of ACS show promise at developing more physiologic, targeted treatments. These developments include modulation of the inflammatory response and new diagnostic modalities. Moreover, these advancements all address the current shortcomings in the diagnostic armamentarium that trauma surgeons can use, and which should lead to more precise methods 
to diagnose ACS and less invasive ways to prevent and treat it. Many of the approaches discussed in this chapter may be used simultaneously, and a multimodality approach to diagnosis and therapy, for example, using tissue ultrafiltration, intermittent plantar compression, small-volume resuscitation, and pharmacologic therapy, may someday be able to supplant fasciotomy for many patients.

\section{References}

1. Janzing HMJ, Broos PLO. Routine monitoring of compartment pressure in patients with tibial fractures: beware of overtreatment! Injury. 2001;32:415-21.

2. Prayson MJ, Chen JL, Hampers D, et al. Baseline compartment pressure measurements in isolated lower extremity fractures without clinical compartment syndrome. J Trauma. 2006;60:1037-40.

3. Whitney A, O' Toole RV, Hui E, et al. Do one-time intracompartmental pressure measurements have a high false-positive rate in diagnosing compartment syndrome? J Trauma Acute Care Surg. 2014;76:479-83.

4. Lawendy A-R, Bihari A, Sanders DW, et al. Compartment syndrome causes systemic inflammation in a rat. Bone Joint J. 2016;98-B:1132-7.

5. Lawendy A-R, Bihari A, Sanders DW, et al. Contribution of inflammation to cellular injury in compartment syndrome in an experimental rodent model. Bone Joint J. 2015;97-B:539-43.

6. Lawendy AR, Bihari A, Sanders DW, et al. The severity of microvascular dysfunction due to compartment syndrome is diminished by the systemic application of CO-releasing molecules (CORM-3). J Orthop Trauma. 2014;28:e263-8.

7. Lawendy A-R, McGarr G, Phillips J, et al. Compartment syndrome causes a systemic inflammatory response and remote organ injury. J Bone Joint Surg Br. 2011;93-B(Suppl III):280.

8. Lawendy AR, Sanders DW, Bihari A, et al. Compartment syndrome-induced microvascular dysfunction: an experimental rodent model. Can J Surg. 2011;54:194-200.

9. Manjoo A, Sanders D, Lawendy A, et al. Indomethacin reduces cell damage: shedding new light on compartment syndrome. J Orthop Trauma. 2010;24:526-9.

10. Garr JL, Gentilello LM, Cole PA, et al. Monitoring for compartmental syndrome using nearinfrared spectroscopy: a noninvasive, continuous, transcutaneous monitoring technique. J Trauma. 1999;46:613-6.

11. Bariteau JT, Beutel BG, Kamal R, et al. The use of near-infrared spectrometry for the diagnosis of lower-extremity compartment syndrome. Orthopedics. 2011;34:178.

12. Shuler MS, Reisman WM, Kinsey TL, et al. Correlation between muscle oxygenation and compartment pressures in acute compartment syndrome of the leg. J Bone Joint Surg Am. 2010;2:863-70.

13. Shuler MS, Reisman WM, Cole AL, et al. Near-infrared spectroscopy in acute compartment syndrome: case report. Injury. 2011;42:1506-8.

14. Shuler MS, Reisman WM, Whitesides TE, et al. Near-infrared spectroscopy in lower extremity trauma. J Bone Joint Surg Am. 2009;91-A:1360-8.

15. Shuler MS, Roskosky M, Kinsey T, et al. Continual near-infrared spectroscopy monitoring in the injured lower limb and acute compartment syndrome. An FDA-IDE trial. Bone Joint J. 2018;100-B:787-97.

16. Schmidt AH, Bosse MJ, Obremskey WT, et al. Continuous near-infrared spectroscopy demonstrates limitations in monitoring the development of acute compartment syndrome in patients with leg injuries. J Bone Joint Surg Am. 2018;100:1645-52.

17. Odland RM, Schmidt AH. Compartment syndrome ultrafiltration catheters: report of a clinical pilot study of a novel method for managing patients at risk of compartment syndrome. $\mathrm{J}$ Orthop Trauma. 2011;25:358-65. 
18. Odland R, Schmidt AH, Hunter B, et al. Use of tissue ultrafiltration for treatment of compartment syndrome: a pilot study using porcine hindlimbs. J Orthop Trauma. 2005;19:267-75.

19. Elliott K (2014) Intramuscular pH: Diagnosing acute compartment syndrome with confidence," in Proceedings of the 2014 London EFORT Conference Trauma Session.

20. Harvey EJ, Sanders DW, Shuler MS, et al. What's new in acute compartment syndrome? J Orthop Trauma. 2012;26:699-702.

21. Agudelo JF, Morgan SJ, Schmidt AH, et al. Management of acute compartment syndrome: randomized clinical trial evaluating a novel compartment syndrome catheter. OTA Annual Meeting, Ottawa, CAN, Oct. 2005.

22. Sanders DW, Chan G, Badhwar A. Hypothermia in compartment syndrome. J Bone Joint Surg Br. 2011;93-B(SUPP III):280.

Open Access This chapter is licensed under the terms of the Creative Commons Attribution 4.0 International License (http://creativecommons.org/licenses/by/4.0/), which permits use, sharing, adaptation, distribution and reproduction in any medium or format, as long as you give appropriate credit to the original author(s) and the source, provide a link to the Creative Commons license and indicate if changes were made.

The images or other third party material in this chapter are included in the chapter's Creative Commons license, unless indicated otherwise in a credit line to the material. If material is not included in the chapter's Creative Commons license and your intended use is not permitted by statutory regulation or exceeds the permitted use, you will need to obtain permission directly from the copyright holder.

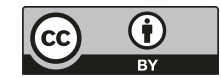

